\title{
PASOS Y TROPIEZOS \\ LAS DIFICULTADES Y LOS LOGROS DE LOS SUJETOS DE LA FORMACIÓN DOCENTE EN EL ABORDAJE DE LA INVESTIGACIÓN EDUCATIVA
}

\author{
Por María del Carmen Cid ${ }^{*}$ \\ Instituto Superior de Formación Docente º 803, Argentina. \\ cidmaria@gmail.com
}

Recibido: 16/09/2016 Aceptado: 07/11/2016

\section{Resumen}

Este artículo refleja un intento de sistematización de las experiencias relevadas durante seis años de desempeño como docente en los Seminarios de Investigación Educativa, en el Instituto Superior de Formación Docente N 803 de Puerto Madryn, Chubut.

Pretende brindar un aporte para la comprensión de cuáles son las dificultades de los y las estudiantes al momento de cursar el Seminario, mediante la identificación de las principales barreras que obstaculizan los procesos de investigación que deben realizar para acreditar la unidad curricular. Por otra parte, se intentan establecer algunas de las principales razones por las cuales surgen las dificultades en el desarrollo de tales procesos, tanto desde la perspectiva metodológica como desde otros aspectos concomitantes que no pueden ser dejados de lado.

Simultáneamente, resulta interesante identificar qué estrategias ponen en juego los y las estudiantes, en orden a superar las dificultades, y cómo van ajustando sus acciones, a lo largo de un recorrido espiralado, hasta poder dar

Licenciada en Educación. Orientación en Gestión Institucional (UNQ). Arquitecta (FADU-UBA). Maestra Normal Nacional (Instituto Sagrado Corazón de Castelar). Profesora del ISFD N 803 de Puerto Madryn. 
respuesta a sus interrogantes iniciales, y arribar a conclusiones que constituyan aportes originales al conocimiento sobre una determinada problemática. En este marco, cobran especial relevancia las estrategias que se despliegan desde el/la docente, con el fin de acompañar este proceso y facilitar el aprendizaje.

Palabras Clave: Dificultades - Logros - Formación docente - Investigación educativa - Vía artesanal de transmisión de saberes

\section{Abstract}

This article showcases an attempt to systematize the experiences collected during six years as a teacher in the Education Research Seminars in the Instituto Superior de Formación Docente $N^{\circ} 803$ from Puerto Madryn, Chubut.

It aims to provide a contribution into understanding which are the difficulties for the students attending the seminars, through the identification of the main obstacles blocking the research processes they must accomplish in order to credit the curricular unit. In the other hand, it also attempts to establish some of the main reasons for the difficulties arising during such processes, from a methodological point of view as well as several other present angles that should not be left aside.

At the same time, it is interesting to identify the strategies displayed by the students in order to overcome the difficulties, and how they adjust their actions along a spiral path until they reach an answer to their initial questions and reach conclusions that constitute original contributions into the knowledge of a given problem. In this framework, the strategies played by the teacher, in order to help and ease the students learning processes, become specially significant.

Keywords: Difficulties - Achievements - Subjects of teacher training - Educational research - Artisan transmission of practical knowledge.

\section{Introducción}

Este artículo presenta un primer intento de sistematización de la experiencia realizada como docente de los Seminarios de Investigación Educativa del ISFD N ${ }^{\circ} 803$ de Puerto Madryn en los últimos seis años, en los Profesorados de Formación Ética y Ciudadana, Inglés y Educación Inicial, y tiene el objetivo de revisar la información disponible surgida del desarrollo de esta experiencia, considerando que compartir algunos interrogantes y potenciales respuestas podría resultar útil a quienes en la actualidad abordan la enseñanza y el aprendizaje de la investigación educativa desde su práctica o como parte de su formación.

Enseñar a investigar es, según sostiene Elsie Rockwell (2009), ejercitarse en una manera de estudiar tanto el presente como el pasado, integrando el 
rigor del análisis documental con la agudeza de la mirada, para describir ambientes y narrar procesos que no se encuentran explícitos en ningún discurso oficial, pero que explican gran parte de lo que está en juego en la educación.

Como docente muchas veces me he preguntado cuál es la mejor manera de explicar qué es hacer investigación cualitativa en el campo de la educación [1], y siguiendo a Scribano (2008) pienso que la única certeza es que no hay recetas, sino apenas la oportunidad de posibilitar una reflexión, para la cual disponemos de diversas alternativas, según sean nuestros interrogantes y nuestros propósitos. Valga entonces la aclaración para reforzar el carácter de aproximación de este trabajo, con la idea de sumar mis propias inquietudes a las de colegas y estudiantes.

En tal sentido, el material de campo de lo que a continuación se expone, son los registros de las producciones realizadas por diez cohortes de las mencionadas carreras, que oportunamente y durante el transcurso de los respectivos seminarios, fueron revisadas por la docente.

Se trabajó con las producciones de treinta y ocho equipos de estudiantes, integrados por una media de cuatro estudiantes cada uno, quienes elaboraron sus proyectos de investigación, concretaron el trabajo de campo y formularon sus informes finales. Tanto el documento del proyecto, como el del informe final, se convirtieron en objeto de revisiones reiteradas por parte de la docente y de los y las estudiantes, a lo largo del término lectivo. La unidad de análisis para esta indagación, es, por lo tanto, cada una de las revisiones realizada.

Una pequeña reflexión respecto de las palabras clave que acompañan el título de este artículo: teniendo en cuenta que al comenzar el seminario los y las estudiantes toman contacto con la cuestión metodológica de la investigación educativa, en general sin otro antecedente a lo largo de su trayecto formativo, entendemos por dificultades (los "tropiezos" a los que alude el título), aquéllas que se verifican en todo proceso de aprendizaje, donde la interacción con un nuevo objeto de conocimiento propone tiempos de apropiación que coexisten con errores, malinterpretaciones o desvíos, que necesariamente forman parte del proceso de construcción cognitiva.

Por otro lado, al hablar de logros (los "pasos" hacia adelante que el título sugiere), es necesario tomar el recaudo de considerar a qué objetivo pedagógico otorgaremos mayor jerarquía. En este caso, se ha priorizado posibilitar un recorrido que deje como saldo la comprensión y apropiación del proceso en sí, arribando a unos resultados que, sin necesidad de ser espectaculares, se despeguen del saber de sentido común. Simultáneamente, ha parecido fundamental propiciar el descubrimiento del placer que puede encontrarse en esta actividad, que propicia el cuestionamiento de las propias prácticas y habilita el camino para transformarlas.

Este artículo se propone exponer con cierto detalle las dificultades que los y las estudiantes encuentran al aprender a investigar, para intentar algunas 
interpretaciones acerca de por qué éstas se convierten en obstaculizadoras del proceso. Finalmente se tratará de explicar cómo se articulan un enfoque de la enseñanza de la investigación educativa con una propuesta didáctica orientada a superar tales dificultades, para alcanzar los saberes necesarios para acreditar.

Sobre los tropiezos

\section{Lo que dicen los datos}

Las revisiones que componen la muestra, ponen en evidencia, a través de los comentarios realizados por la docente en cada una de ellas, una serie de observaciones que pueden ser clasificadas en tres grandes grupos, los cuales tienen diferente peso relativo en la totalidad del conjunto. Estos grupos, discriminados en el GRÁFICO I, dan cuenta de observaciones:

- Relacionadas con el uso de la lengua escrita como código (28 \%).

- Relacionadas con las características del texto producido (13\%).

- Relacionadas con aspectos metodológicos de la investigación (59\%).

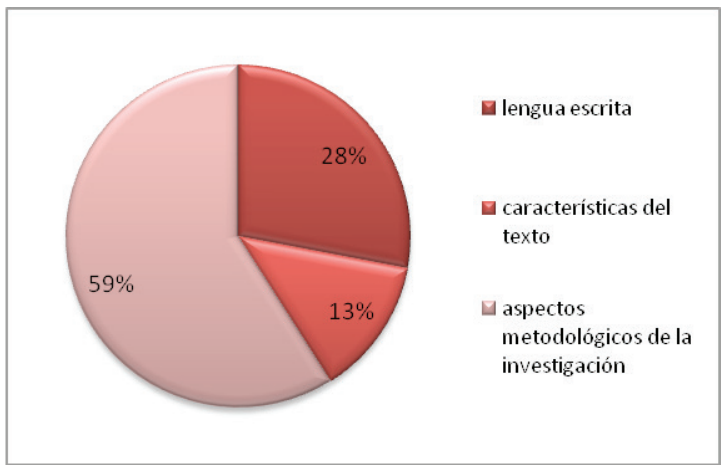

GRÁFICO I - TIPO DE OBSERVACIONES

EI GRÁFICO II, que se incluye a continuación, expone las observaciones que hacen referencia a dificultades en el uso de la lengua escrita como código (28 \% en el Gráfico I). Considerándolas en su totalidad, discrimina qué tipos de fenómenos constituyen dichas dificultades, y con qué valores se hacen presentes. 


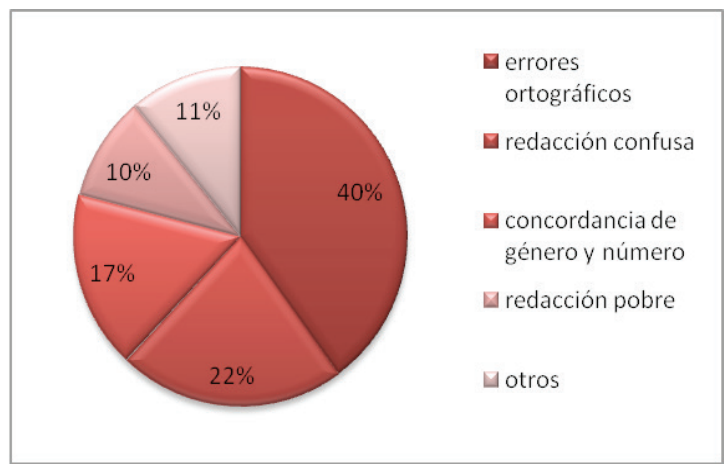

GRÁFICO II - OBSERVACIONES REFERIDAS A LA LENGUA ESCRITA COMO CÓDIGO

Los errores de ortografía son el fenómeno más relevante, representando un $40 \%$ del total de dificultades vinculadas al uso de la lengua escrita. Le sigue la redacción confusa, que obstaculiza la comprensión del mensaje elaborado, con un $22 \%$ del total. La ausencia de concordancia de género y número tiene un peso relativo del $17 \%$ del total del grupo. La pobreza en la redacción (uso de vocabulario inadecuado o escaso, presencia de reiteraciones, sustituciones u omisiones de términos) aparece con un valor del $10 \%$. Otras dificultades (entre ellas la ausencia o uso incorrecto de mayúsculas o de signos de puntuación) representan, en conjunto, un $11 \%$ del total.

En el GRÁFICO III, presentado a continuación, se exponen las dificultades que tienen que ver con las características del texto producido, aquéllas que aparecen en el Gráfico I: Tipos de observaciones, con un peso relativo del $13 \%$.
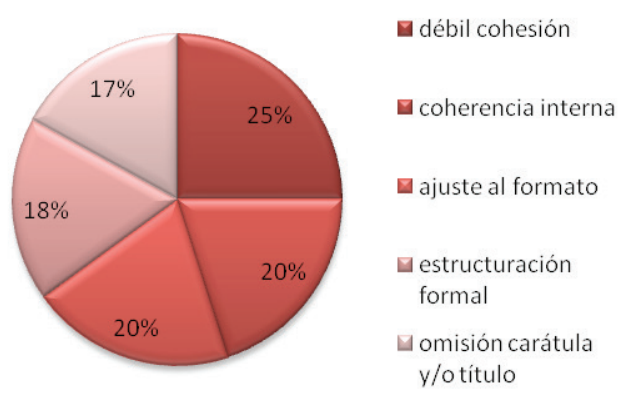

GRÁFICO III - OBSERVACIONES REFERIDAS A CARACTERÍSTICAS DEL TEXTO

Aparecen, con un porcentaje del $25 \%$, los aspectos relacionados con la cohesión textual, que se ponen en evidencia en la ausencia de conectores adecuados, el uso cambiante de tiempos verbales o la inestabilidad de los 
sujetos de la enunciación. Asimismo, en un $20 \%$ de los casos, se constata debilidad en la coherencia interna del documento (reflejada en afirmaciones contradictorias), bajo grado de articulación entre sus componentes, o la inexistencia de un eje claro de progresión temática. También con un $20 \%$ aparecen cuestiones que se conectan con un débil ajuste al formato textual, tanto en el proyecto como en el informe final.

Finalmente, se observan cuestiones de carácter más convencional, por ejemplo en la estructuración formal del texto, en la que se consideran aspectos tales como la organización en párrafos de extensión y contenido adecuados a lo que se expone, el inicio y cierre correcto de comillas, la estabilidad del interlineado o la tipografía a lo largo de todo el documento, así como la presentación convencional de citas bibliográficas literales mayores de cuarenta palabras. El peso relativo de este tipo de observaciones es de un $18 \%$ del total del grupo. La omisión de la carátula y del título de la investigación, con una presencia del $17 \%$, es otro de los aspectos afines a esta clase de observaciones.

EI GRÁFICO IV refleja aquellas observaciones referidas a aspectos metodológicos de la investigación, que en el Gráfico I representan un $59 \%$ del total de observaciones realizadas. Vale resaltar la contundencia de su presencia dentro del conjunto global de los datos, e indicar que, por la complejidad y diversidad de las cuestiones que contemplan, ha resultado conveniente desagregarlas en tres subgrupos de análisis, vinculados a los tipos de revisiones en las que se hacen presentes.

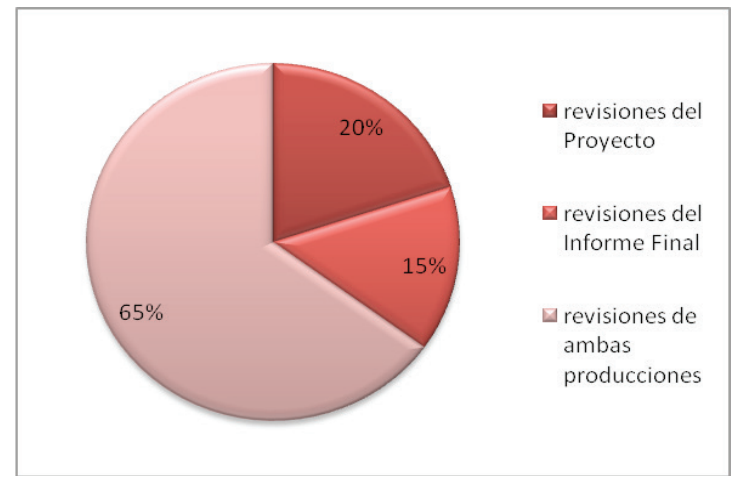

GRÁFICO IV - OBSERVACIONES REFERIDAS A ASPECTOS METODOLÓGICOS

Estos subgrupos son:

1. Observaciones con presencia en las revisiones del Proyecto de Investigación (20 \% del total del grupo).

2. Observaciones con presencia en las revisiones del Informe Final (15 $\%$ del total del grupo). 
3. Observaciones que aparecen indistintamente en ambos tipos de documentos (65\% del total del grupo).

Cada uno de estos desagregados habilita una indagación detallada de los tipos de dificultades que las sucesivas producciones ponen en evidencia a medida que avanzan en su construcción.

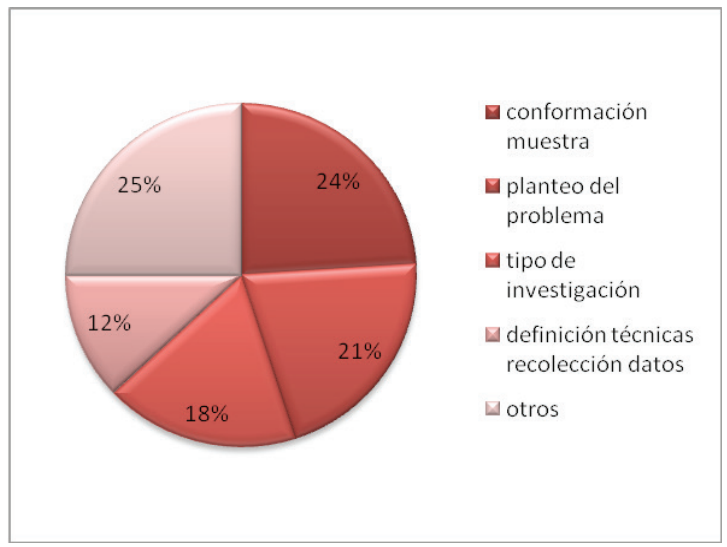

GRÁFICO V - OBSERVACIONES CON PRESENCIA EN EL PROYECTO

En el primer subgrupo, detallado en el GRÁFICO V: Observaciones consignadas en las revisiones de los avances del proyecto, la mayor proporción se registra en las imprecisiones al definir las muestras o la omisión de criterios de conformación (24\%). Le siguen las observaciones referidas a la debilidad con la que se plantea el problema y los interrogantes de investigación $(21 \%)$, para continuar con las que hacen alusión a la definición incompleta del tipo de investigación (18\%), ya sea porque se omite explicitar el paradigma o se clasifica desde un único criterio. Se suman las observaciones relacionadas con la elección de las técnicas de recolección de datos (12\%), que resultan confusas o insuficientes. Otro tipo de observaciones (25\%), puntualizan la omisión de las categorías de análisis, la elaboración de un marco teórico débilmente articulado con la problemática elegida, confuso o pobre en contenido conceptual, la confusión entre técnicas e instrumentos, y la ausencia del apartado relacionado con la justificación del proyecto. 


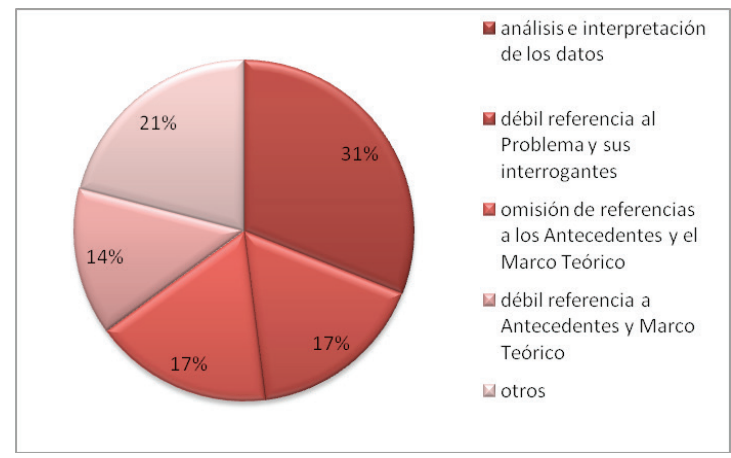

GRÁFICO VI - OBSERVACIONES CON PRESENCIA EN LOS INFORMES FINALES

El segundo subgrupo, analizado en el GRÁFICO VI: Observaciones con presencia en los informes finales, registra, con un $31 \%$, señalamientos vinculados a la pobreza del proceso de interpretación de resultados y de la elaboración de conclusiones, debido a que el análisis de los datos no se compromete con el esfuerzo intelectual de descubrir lo subyacente a una lectura lineal, sosteniéndolo a través de una argumentación sólida. Le siguen, con un $17 \%$, la débil referencia al problema de investigación y sus interrogantes, así como a los antecedentes y conceptos teóricos que le dieron sustento epistemológico, también con un valor del $17 \%$. En menor proporción, se plantea, con un $14 \%$, la escasa contundencia al presentar los datos obtenidos, desatendiendo las referencias claras a las técnicas que les dieron origen y sin citas textuales de los dichos de los informantes como recurso de respaldo de la exposición, con la consecuente debilitación del hilo argumentativo. Finalmente, otras observaciones del orden del $21 \%$ del total, consideran tanto la ausencia de relato del proceso y ajustes a los que obligaron las circunstancias del trabajo de campo, como la no recuperación de los supuestos iniciales para contrastarlos con los resultados obtenidos, y la ausencia de las categorías de análisis como organizadoras del discurso argumentativo.
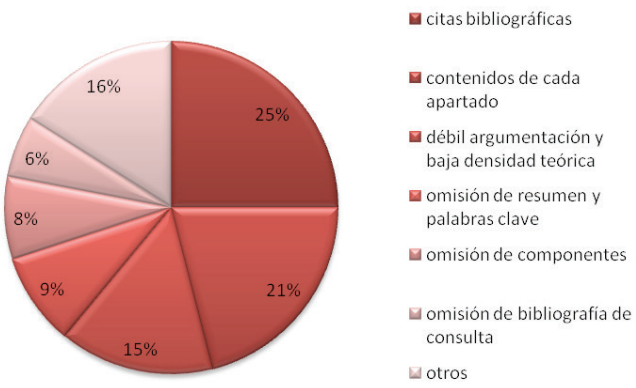
El tercero de los subgrupos que propone el GRÁFICO VII: Observaciones con presencia en ambos documentos (proyecto e informe final), indica que un $25 \%$ de los y las estudiantes no realizan las citas respetando las normas en uso y consignando datos completos, tanto aquéllas incorporadas al texto, como las incluidas en el apartado de bibliografía. Le sigue, con un $21 \%$, la confusión respecto al contenido de cada uno de los componentes del documento (suelen incorporar materiales auxiliares: fichaje de textos en el caso del estado del arte o listados de potenciales preguntas de investigación descartadas en el planteo del problema) o la alteración del orden lógico en que deben sucederse dichos componentes (planteo de objetivos previo al desarrollo del marco teórico; elección de las técnicas de recolección de datos antes de plantear el problema y sus preguntas, por ejemplo). Con un $15 \%$ se evidencia la dificultad para lograr un nivel argumentativo potente, sustentado en un denso respaldo teórico. Un $9 \%$ de los trabajos omite incluir el resumen y las palabras clave. En proporción del 8 \%, alguno de los componentes de ambos documentos es suprimido. El apartado de referencias bibliográficas completas suele ser tardíamente incorporado e incluso omitido (6\%). Otras observaciones, con un peso del $16 \%$ en total, dan cuenta de inconsistencia interna del documento (falta de articulación entre sus componentes), omisión de fuentes, descuido de la obligación de preservar la confidencialidad de los informantes o las instituciones en las que se lleva a cabo el trabajo de campo, escasas estrategias de vigilancia epistemológica que garanticen la confiabilidad y validez de los resultados (la triangulación de fuentes, técnicas e investigadores, consideraciones sobre la reflexividad de investigadores e informantes, o la duda sistemática ante proposiciones que responden a estereotipos). Es además habitual que los conceptos clave tengan escasa presencia a lo largo del documento.

\section{Sobre los logros de las y los estudiantes}

Acerca de los logros que van obteniendo los y las estudiantes durante el desarrollo del seminario, contamos con el dato cuantitativo de la relación existente entre la cantidad de inscritos e inscritas que inician la asistencia al mismo y la cantidad de estudiantes que lo acreditan en los plazos estipulados por las normas académico-institucionales.

Los seminarios de investigación no se acreditan en carácter de libre, la conservación de la regularidad obliga a obtener la promoción directa al fin del ciclo lectivo, o posteriormente, en las mesas de examen. De no lograr esta acreditación, el camino obligatorio es volver a cursar el seminario.

Estudiantes que acreditan con promoción directa son aquéllos que finalizan el término lectivo con una producción que se ajusta a las características esperadas.

Promovidos en mesa de examen son los y las estudiantes que no han perdido su condición de regulares, pero han llegado a la finalización del ciclo lectivo con producciones que no se ajustan a las características esperadas. Deben acreditar 
la unidad curricular en instancias de examen, reformulando los aspectos que les hayan sido indicados.

No promocionados son quienes por diferentes razones pierden su regularidad (no cumplen con las tareas pautadas u obtienen resultados extremadamente pobres) y los que abandonan el seminario por razones ligadas a las vicisitudes que atraviesan su trayectoria formativa o a causas de orden personal o familiar que la interfieren.

Según lo expresado por el GRÁFICO VIII: Resultados totales del cursado del seminario, una amplia mayoría de los y las estudiantes superan las dificultades en el abordaje de la investigación educativa, al finalizar el término lectivo, o posteriormente, en mesas de examen, concretando producciones que tienen las características requeridas para acreditar.

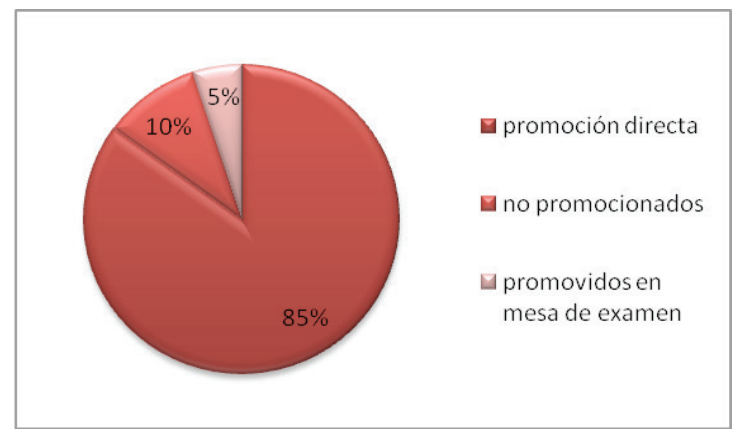

GRÁFICO VIII - RESULTADOS TOTALES DEL CURSADO DEL SEMINARIO

En efecto, un $85 \%$ del total de la matrícula integrada por las diez cohortes que lo cursaron durante los seis años en los que se focaliza este estudio, lograron promocionarlo directamente al finalizar el ciclo lectivo. Un $5 \%$ de los y las estudiantes pudieron acreditar en mesa de examen, mientras que un $10 \%$ debieron volver a cursar.

De lo expuesto, y más allá de las dificultades que han sido descritas, pareciera posible inferir que los resultados, en relación con los propósitos priorizados por la docente para la unidad curricular, dan cuenta de procesos en los que, mayoritariamente, los y las estudiantes logran apropiarse de los saberes requeridos para iniciar la experiencia de convertirse en investigadores de sus propias prácticas.

\section{A la búsqueda de sentidos}

Como ya lo ha expresado Rosana Guber (2013) la investigación, sobre todo la de estirpe cualitativa y con un enfoque etnográfico [2], tal como lo establecen los diseños curriculares en Chubut, se torna más sabia cuanto más nos permean los sujetos que son protagonistas de la realidad estudiada; más 
profunda, cuanto más evidente nos resulta nuestra propia ignorancia. No discurre en línea recta, aplicando consignas teóricas sobre el material empírico recolectado, sino que la dinámica entre trabajo teórico y trabajo de campo produce procesos paralelos y entrelazados, que se imbrican dialécticamente, tornándose cíclicos, recurrentes y espiralados.

Probablemente esta condición de incertidumbre que genera un ir y venir entre lo que se piensa, lo que se proyecta y lo que finalmente se hace, es lo que más desestabiliza a quienes abordan por primera vez la experiencia de la investigación, y explica en parte lo expresado en el Gráfico I, en el que las observaciones vinculadas a la cuestión metodológica adquieren un peso relevante. El seminario plantea un devenir en el que las acciones casi nunca son definitivas, al menos durante un buen período de su desarrollo, sino que el conjunto se plantea como un todo en permanente articulación.

Sin embargo, hay que prestar atención al hecho de que las dos categorías restantes, vinculadas a la escritura de los textos, suman un porcentaje importante de observaciones en relación con el que detentan las cuestiones metodológicas. Lejos de tratarse de cuestiones formales, tienen directa incidencia en el proceso intelectual de la investigación, ya que tanto el giro lingüístico, en el sentido en que ha sido abordado por autores como Bergmann (1953), Rorty (1990), Foucault (1997), Derrida (1989) y otros, así como la hermenéutica [3], concebida como el arte o la teoría de interpretar textos considerando presuposiciones y pre-comprensiones, aspectos semióticos, verbales y no verbales, tal como lo expresan entre otros Gadamer (1998) y Vattimo (1995), nos enseñan que el lenguaje construye nuestro pensamiento.

En ese sentido, aquellas dificultades directamente relacionadas con el uso de la lengua escrita, inciden en nuestra percepción de la realidad, obstaculizan nuestra capacidad de construir significados y limitan, por ende, nuestro pensamiento.

Sin embargo, estos aspectos de la producción, que refleja el Gráfico II, no suelen preocupar demasiado a los y las estudiantes. En muchas oportunidades, los errores de ortografía, la ausencia de concordancia, el uso correcto de signos de puntuación o de mayúsculas, son aspectos indicados desde las primeras revisiones sin que los equipos ajusten su producción hasta último momento.

Los errores ortográficos tienen menor peso relativo que la suma de los demás aspectos considerados en el párrafo precedente, a los que se añade la redacción confusa o pobre. Cabe señalar que todas estas cuestiones inciden sobre la inteligibilidad de lo que se expresa, sobre la estructuración del propio pensamiento y sobre la potencial función comunicativa.

El Gráfico III nos sitúa en la cuestión propiamente textual, considerando al texto como instrumento que permite la construcción epistemológica del objeto, así como la reflexión propia del proceso de investigación y la posibilidad de generar interpretaciones de lo que se observa. 
Es importante destacar que siendo tanto la cohesión textual, como el ajuste al formato, aspectos que hacen a la coherencia interna del texto y a su posibilidad de ser interpretado, no resulta sorprendente encontrar que estos tres tipos de dificultades registradas en las revisiones constituyen el núcleo más duro de los obstáculos para la producción. Los dos ítems restantes de este tramo del análisis, la estructuración formal del texto, la falta de carátula y/o título, están vinculados a las condiciones de presentación.

A continuación corresponde abordar el tema de las observaciones relacionadas con aspectos metodológicos de la investigación.

Con el Gráfico IV abordamos ese tipo de observaciones, para desagregarlas en tres subgrupos: a) las que tienen presencia en las revisiones de proyectos; b) aquéllas que aparecen en los informes finales; y c) las observaciones con presencia en ambos tipos de documentos.

La primera cuestión a señalar es el predominio absoluto de observaciones del tercer grupo (65\%) sobre los dos primeros consignados (35\%), lo que podría estar indicando que algunas de las dificultades que encuentran los y las estudiantes al abordar la experiencia, están muy vinculadas a particularidades que hacen a la actividad de investigar, en detrimento de aspectos específicos propios de la producción de los textos que dan cuenta de cada una de las etapas que supone esta actividad.

Efectivamente, el cotejo en detalle de cada uno de estos subgrupos (Gráficos V, VI y VII) expresa un predominio de dificultades vinculadas a la dimensión estratégica del proyecto en el primer caso; una mayor proporción de observaciones relacionadas con la interpretación de datos y atribución de significados, articulada con aspectos de la dimensión epistemológica del proyecto, en el caso del informe final; $y$ en el tercer caso, aspectos que tienen que ver con la mirada comprensiva y global sobre lo que significa hacer investigación y sobre la apropiación y exigencias de la manera de llevarla a cabo. Lo indicado parecería expresar una lógica compatible con la situación de estar iniciándose en un territorio poco conocido y complejo, donde las pocas certezas y la ausencia de preconceptos acerca de lo que se va a hallar son consideradas más virtudes que deficiencias. Tal circunstancia provoca ansiedad en los que transitan la experiencia, quienes muy frecuentemente pasan del entusiasmo al desaliento, a la confusión, a la creencia de que no acaban de aprender cómo se hace, al agobio de creer que deben empezar de nuevo y que nada de lo hecho sirve. Es función del acompañamiento docente desarmar esta mirada negativa y convencer de que lo que ocurre es parte del proceso e implica haber dado pasos hacia adelante.

En el caso del proyecto (Gráfico V), luego de la trabajosa construcción epistemológica, que demanda sustento teórico, y a las consideraciones de tipo abstracto vinculadas a la problemática en análisis, los estudiantes encuentran que es necesario tomar una serie de decisiones estratégicas y prácticas que 
les permitirán sumergirse en el campo y desarrollar actividades concretas. Estas decisiones responden a criterios múltiples y complejos, ya que, además de resultar absolutamente articuladas con el problema de investigación y sus interrogantes, así como establecer las categorías que permitirán organizar el análisis, deben ajustarse a condiciones de viabilidad, disponibilidad de tiempos y espacios, selección adecuada de informantes y técnicas, construcción de instrumentos pertinentes, y demás requisitos.

Aunque con un peso relativamente menor, también se registran dificultades relacionadas con aspectos epistemológicos: todos sabemos lo difícil que es definir un problema de investigación y poder plantearse las preguntas relevantes al respecto, así como determinar el encuadre teórico y los conceptos fundamentales que iluminarán nuestra mirada sobre el mismo, proporcionando el recorte correspondiente.

Las observaciones formuladas a los informes finales (Gráfico VI), en cambio, están dando cuenta fundamentalmente de tres aspectos:

a) lo difícil que resulta superar la lectura superficial de la realidad para interpretar lo que subyace a un recorte de la misma;

b) el poder articular la ida y la vuelta, a y desde lo proyectado inicialmente, sin perder los interrogantes ni los objetivos iniciales, pero logrando incorporar aquello emergente y no previsto de la experiencia de campo, aún a costa de desechar supuestos y concepciones previas acerca de la problemática; $y$

c) el esfuerzo intelectual que significa construir un relato que, sin pretensión de universalidad, proponga la conceptualización de lo observado empíricamente, promoviendo una comprensión más profunda y compleja sobre la problemática elegida. Las tres cuestiones hacen a lo que Clifford Geertz (1987) caracterizaba como descripción densa; no porque fuera un texto de lectura tediosa, sino para referirse a la labor del investigador en la reconstrucción y explicitación del marco interpretativo con el que las personas (en este caso quienes habitan los entornos educativos cotidianamente) dan sentido al mundo.

Es muy frecuente que estas dificultades, sumadas a la falta de tiempo, deriven en interpretaciones pobres y conclusiones estereotipadas que no logran superar una mirada superficial sobre los problemas abordados, apegada a la linealidad del saber de sentido común, desmereciendo un proceso que pudo haberse desarrollado con riqueza hasta ese momento. En este caso, hay que reorientar el trabajo de los grupos y destacar la relevancia de esta etapa de la investigación, la que demanda mayor esfuerzo intelectual, y que, según Rockwell (2003), constituye su momento más difícil. Presupone un procedimiento insustituible, el trabajo sobre los textos producidos: leer, releer y releer; interpretarlos desde varios ángulos, anotar y anotar sobre anotaciones, relacionar, dudar, volver a relacionar, escribir y romper, todo ello hasta encontrar cómo 
encajan algunas piezas del rompecabezas.

Por último, la mirada sobre el Gráfico VII nos sitúa sobre aspectos que dan cuenta de dificultades que aparecen a lo largo de todo el proceso de investigación y que nos hablan de que no resulta fácil comprender globalmente cómo es ese proceso, a qué procedimientos se recurre, con qué lógicas, y cuáles son las exigencias que la comunidad científica plantea a la actividad.

Si bien la inmersión en el campo se produce tempranamente, ya que los y las estudiantes suelen elegir investigar en las escuelas a las que asisten por requisitos vinculados a los espacios de práctica profesional docente, suele ocurrir que no se perciba que existe una lógica interna para cada una de las producciones. Vale decir: no es posible desarrollar un encuadre teórico sin haber definido un problema o sin consultar bibliografía; no podemos establecer objetivos sin haber revisado antecedentes. Tampoco podemos elaborar conclusiones sin haber planteado cuidadosamente los datos y sin haberles otorgado un sentido. No es posible producir teoría sin argumentar y prescindiendo de citar autoridades reconocidas que respalden nuestras afirmaciones. O sea: pese a que hay un permanente ir y venir entre componentes de cada documento, éstos mantienen entre sí relaciones que nos plantean ciertas exigencias, cronologías y prioridades.

Mención aparte merece el tratamiento de la bibliografía. Lejos de constituir una cuestión menor, la explicitación de las fuentes es lo que hace la diferencia entre el plagio y el reconocimiento de los hallazgos originales de otros investigadores. Los y las estudiantes tienen ciertas dificultades para convencerse de ello. La generosa disponibilidad del conocimiento es una de las características de la ciencia; el uso poco cuidadoso de las producciones ajenas es inadmisible. Efectuar las citas y consignar las referencias bibliográficas de acuerdo con la convención académica es un rasgo propio de la actividad; sin embargo los y las estudiantes suelen tener una gran resistencia a concederle la importancia que merece.

El descuido de la obligación de confidencialidad, así como la consideración de estrategias de vigilancia epistemológica [4] que garanticen la validez y confiabilidad de los resultados a lo largo del proceso, mediante la triangulación de fuentes y técnicas, la atención de la reflexividad de los investigadores y de los informantes, la consideración de la historicidad del fenómeno en estudio, la actitud desconfiada frente a discursos estereotipados acerca del mismo, son también dificultades que aparecen durante el proceso, atribuibles al carácter iniciático de la misma y la necesidad de tiempos, que no siempre coinciden con los que plantea el calendario escolar, para la apropiación de un saber que, según se profundizará en apartados posteriores, consiste en bastante más que comprender y apropiarse de una teoría.

Cabe ocuparnos del Gráfico VIII, que refiere aspectos cuantitativos de la acreditación del seminario por parte de los y las estudiantes, sin aportar 
detalles respecto de la calidad y características del recorrido que realizan al cursarlo. Tan solo informa que éstos, en su mayoría, arriban a resultados adecuados para acreditar. Significa que los grupos obtienen, en ocasiones, hallazgos interesantes, pero siempre con un crecimiento cualitativo relevante en la destreza para abordar la actividad.

Interesa destacar que es frecuente que, luego de los primeros encuentros en los que se plantean aspectos vinculados con el encuadre general del seminario y la conformación de los equipos de investigación, la evaluación y el debate relacionados con los temas de interés para cada uno de ellos, la búsqueda de las preguntas relevantes que justifiquen el proceso de investigación y concreten el recorte, así como el buceo y registro de antecedentes, que suele provocar cambios y ajustes en lo precedentemente realizado, se produzca un momento de cierta inercia que debe ser superado por los y las estudiantes para sumergirse de lleno en la tarea. Una especie de horror al vacío, el toparse con la constatación de que hay que empezar a hacer. Y este empezar a hacer tiene mucho que ver con intercambiar puntos de vista, preguntar y preguntarse, leer a otros. Pero sobre todo tiene que ver con comenzar a escribir.

Tal como lo menciona Marta Zamero (2013), hay dificultades para asumir que la escritura es una de las tareas habituales de los equipos de investigación, que acompaña tempranamente el proceso, desde las instancias de planificación, textualización y revisión, y que sólo a través de versiones parciales para ser revisadas por los miembros del equipo y por quien enseña, es posible hacer correcciones y reelaboraciones que hacen a la calidad de la producción. Los equipos que comprenden esto más tempranamente son los que se dan mayores oportunidades de logro: documentan sus datos, organizan su tarea y su comunicación, profundizan el conocimiento y lo objetivan, al tiempo que generan instancias metacognitivas que acrecientan su capacidad de llevar adelante el proceso con mayor autonomía.

A su vez, los y las estudiantes deben leer, ya que la escritura es producto de mucha y muy buena lectura. El acceso al discurso científico propio de un determinado campo oficia de modelo inspirador que permite apropiarse del modo particular de comunicar, del vocabulario específico y del dominio de sus géneros.

Leer es, en cualquier caso, enlazar un discurso nuevo con el discurso del texto.... [...] La interpretación aproxima, iguala, hace que lo extraño resulte contemporáneo y semejante, es decir, convierte en algo propio lo que, en un principio, era extraño (Ricoeur, citado por Rockwell; 2009, p. 87).

El doble ejercicio de la lectura y la escritura, es, desde este punto de vista, la mayor contribución a los logros de los y las estudiantes en el recorrido que plantea el seminario. 


\section{Para cerrar: algunas conclusiones provisorias}

A modo de síntesis, recordemos que este estudio ha tomado como objeto los procesos que realizan los y las estudiantes de la formación docente cuando por primera vez abordan sistemáticamente el aprendizaje de la investigación educativa. Tales procesos conllevan el tener que enfrentar dificultades y poner en juego estrategias que permitan superarlas para alcanzar resultados satisfactorios en un proceso completo de investigación. Algunas de estas dificultades son las propias del abordaje de cualquier campo de conocimiento que resulte desconocido; pero hemos visto que en el caso de la investigación educativa adquieren características particulares, por cuanto exigen la combinación de saberes teóricos y prácticos, en los que se juegan cuestiones epistemológicas, metodológicas y axiológicas que pueden actuar como barreras en el recorrido de quienes aprenden, y a la vez demandan una fuerte articulación con la propuesta didáctica de quien enseña.

Respecto de las dificultades, hemos establecido que en gran parte están relacionadas con aspectos metodológicos de la investigación, pero profundamente imbricadas con la necesidad de producir escrituras. Los y las estudiantes se encuentran en situación de elaborar su propio discurso sobre un determinado fenómeno. En el caso del seminario, este discurso es un discurso escrito, nutrido de voces diversas, en el que se articulan dos dimensiones: la competencia que tienen los y las estudiantes, como usuarios y usuarias del código, y su capacidad de convertirlo en instrumento para simbolizar la realidad. Integrar todos estos planos supone un recorrido de gran complejidad. Facilitar ese proceso integrador es resorte de quien enseña, y el diseño de la propuesta didáctica para la unidad curricular debe tener en cuenta lo precedentemente expresado.

Estos tres aspectos, apropiación de la metodología, prácticas de escritura y propuesta didáctica, que hemos desglosado a efectos exclusivamente expositivos, en la realidad se articulan permanentemente, constituyendo un hacer que se focaliza en la tarea de cada uno de los grupos de estudiantes, donde la búsqueda y comprensión de los aportes teóricos, la transmisión de estrategias y saberes prácticos, la provisión de modelos y el seguimiento crítico y orientador de los avances en las producciones, responde a una forma de concebir la enseñanza de la investigación educativa a la que denominaremos vía artesanal, sobre la cual nos explayaremos más adelante.

El análisis de los aspectos metodológicos evidencia que tempranamente, para los y las estudiantes, es necesario ponerse a hacer. Eso significa, en los términos de Guber (2013) dejarse permear por los saberes de los sujetos de la realidad en estudio, articulando dialécticamente aportes teóricos previos y en permanente interacción con los datos que se recogen en el trabajo de campo. Tales apropiaciones se juegan en el espacio que queda entre la comprensión global y abstracta de la totalidad del proceso de investigación y las prácticas 
concretas de las que la investigación se sirve para operar en el campo de la realidad. La incertidumbre, la necesaria descentración y la dialecticidad de este proceso suman complejidad al recorrido de aprendizaje.

El abordaje de la dimensión epistemológica del proyecto conecta a los y las estudiantes con la lectura de fuentes que les permitirán una construcción teórica que no es el simple registro de lo que afirman los autores consultados, sino una reelaboración propia del equipo, ajustada al recorte elegido del fenómeno a estudiar. En términos de Ricoeur (citado por Rockwell; 2009), es preciso hacer que lo extraño resulte contemporáneo y semejante; convertir en algo propio lo que, en un principio, era extraño. Estas muchas y buenas lecturas requieren el intercambio con el docente para acompañar su selección, su comprensión y ajuste al problema planteado y sus preguntas. A su vez, generan las primeras demandas de escritura: comenzar a ensayar el planteo de esta dimensión epistemológica, elaborando los diferentes apartados del documento, y simultáneamente, iniciar la toma de notas de campo, registrando observaciones que por diferentes razones resulten relevantes durante el proceso de inmersión.

La dimensión estratégica del proyecto, sitúa a los y las estudiantes en la necesidad de tomar decisiones concretas que permitirán desarrollar el trabajo de campo de manera consistente con el desarrollo epistemológico formulado. Aquí las dificultades están ligadas a la capacidad de prever y conciliar aspectos tales como la pertinencia de la conformación de las muestras, el ajuste de las técnicas a los interrogantes, la definición de categorías de análisis y el diseño de instrumentos de recolección de datos, con aspectos vinculados a la distribución de tiempos de trabajo o la viabilidad de las acciones a ejecutar. Nada de ello resulta sencillo. Sobre todo, bajo la premisa de no atarse excesivamente a lo programado, dado que, tal como lo expresa Rockwell (2009), para lograr el conocimiento, la experiencia de campo debe transformar al etnógrafo. Éste no va al campo a confirmar lo que ya creía saber, sino para construir nuevas miradas sobre las realidades ajenas de maestros, estudiantes y comunidades educativas.

Sin embargo, la experiencia no termina en el trabajo de campo, sino que culmina con la producción de representaciones textuales de la realidad estudiada. El informe final es un texto que redactan los grupos a partir de sus trabajos de campo, en el que se privilegia la descripción analítica concentrada, y a la vez detallada, de una selección de lo observado y escuchado en el campo, ordenada y articulada de tal manera que apoye el argumento de fondo y lo articule con la conceptualización teórica. Cuando los grupos encaran la tarea de escribir el informe final, desde el punto de vista metodológico, la dificultad más grande reside en el proceso del análisis cualitativo, el momento de la interpretación de los datos empíricos y su confrontación con los supuestos iniciales y las referencias teóricas del marco. Entra aquí en juego la complejidad de los 
procesos de simbolización y atribución de significado, tal como lo consideraron autores ya citados (Derrida, 1989; Gadamer, 1998; Rorty, 1990; Vattimo, 1995). En el decir de Rockwell (op. cit.), el proceso mental no recorre un camino lógico y pautado. Las intuiciones y las corazonadas cobran importancia, y a los y las estudiantes les cuesta darles lugar. Es necesario superar la tentación de encaminarse por el camino corto y cómodo que lleva a comprobar que se verifican los supuestos iniciales, para realizar un proceso intelectual que establezca categorías más sutiles que las ya dadas por el saber de sentido común y la teoría existente y disponible abordada, para buscar mayor validez en los nexos entre los conceptos y los referentes empíricos, y proponer una relación profunda con la teoría que respalda el estudio. Las varias maneras de mirar el proceso social estudiado, generan en los y las estudiantes una tensión cuya única salida es la transformación de los marcos de interpretación y de análisis previos a la experiencia. Fácil es imaginar las dificultades que estas exigencias plantean a los alumnos y alumnas, exigencias que, por otra parte, son motivo de importante esfuerzo intelectual para cualquier investigador. Pero además de todo lo mencionado, hay que escribirlo.

Al abordar las dificultades que se presentan con la escritura, es necesario consignar que los y las estudiantes tienen, en general, escasas experiencias previas de escritura de textos de las características de los que nos ocupan, y que un peso relevante dentro del proceso señalado lo adquiere la familiaridad y la competencia con la que los equipos de investigación resuelven la producción de textos, ya que: "... partimos de la conceptualización de la escritura como proceso intrínsecamente relacionado con la investigación." (Zamero; 2013; diapositiva 4).

En tal sentido, los sucesivos avances de los equipos están atados a la producción de materiales escritos, aún cuando éstos tengan carácter provisorio y estén destinados a ser revisados y reelaborados, ya que el propio proceso auto-regulado por quienes investigan, así como las orientaciones que puede brindar el o la docente, no se efectúan sobre lo que se cuenta oralmente, sino fundamentalmente sobre lo que se va escribiendo. El detalle concreto de cuáles son estas dificultades ha sido extensamente desarrollado en apartados anteriores, y la propuesta didáctica de quien enseña se articula con ellas mediante la lectura detenida de las producciones, la mención pormenorizada de aspectos resueltos con solvencia y densidad teórica, la provisión de modelos que faciliten la comprensión de los ajustes que se requieren, la presentación de ejemplos y la realización de sugerencias, así como la apertura oportuna de instancias de esclarecimiento conceptual. Si esta estrategia didáctica también se plasma por escrito, si quien enseña también se asume como escritor, los equipos de investigación ven facilitada la dinámica de revisión, ajuste y reformulación, pudiendo ir dando respuesta a multiplicidad de aspectos vinculados con lo que se les señala.

Finalmente, y centrándonos en este caso en la propuesta didáctica, cabe efectuar algunas consideraciones acerca de qué es enseñar a investigar en 
ciencias humanas y sociales, entre ellas las educativas, y qué presupuestos sostienen dicha didáctica. En primer lugar, comprender que, desde una mirada profundamente pedagógica, acompañar el aprendizaje de la investigación educativa no es iniciar una trayectoria que predetermina rutas y modelos, sino generar un espacio en el que quien enseña y quienes aprenden van configurando un camino irrepetible, atravesado por cruces, encuentros y desencuentros, andamiajes de sostén y espacios de crecimiento gradual de la autonomía.

Enseñar a investigar es un proceso complejo y una actividad que presenta múltiples y variadas aristas (Sánchez Puentes; 2014). En su enseñanza concurren operaciones relativas a lo que se enseña al enseñar a investigar y cómo se enseña a investigar. Estas operaciones constituyen dos prácticas distintas, ya que en un caso están vinculadas a producir conocimiento nuevo, y en el otro, a la práctica de enseñar a producirlo. La primera de las operaciones tiene que ver con el oficio de investigador; la segunda, con el de pedagogo. Estos dos perfiles se conjugan en una única persona que se convierte en el encuentro de dos quehaceres que, por separado, son altamente complejos. Tampoco hay una única manera, universal o general, de enseñar a investigar. La enseñanza del quehacer científico educativo está amarrada a la forma de producir conocimiento en dicho campo.

No se enseña a investigar de la misma manera a un estudiante de filosofía, como a uno de sociología, a un físico o a un docente. Pensar la metodología de la investigación social defendiendo una perspectiva general y lógico-formal del quehacer científico, es desvirtuar la generación de conocimiento legítimo y pretender presentar de un modo aséptico lo que en realidad está situado y tiene una perspectiva social y humana.

Tampoco se enseña a investigar en abstracto, repitiendo procedimientos únicos para producir conocimiento. Las estrategias diversificadas introducen invención, incertidumbre, innovación y cambio, que no significan, de manera alguna, descartar el rigor y la disciplina. Investigar es un saber práctico, un saber hacer algo: producir conocimiento. $Y$ los saberes prácticos se transmiten práctica y artesanalmente.

La vía artesanal [5] no es la única manera de enseñar a investigar, pero parece resultar la forma en que se logran mejores resultados. Y por vía artesanal entendemos la comunicación directa entre maestro y aprendiz en ocasión de la transmisión de un oficio. Se aprende a investigar al costado de alguien más experimentado, haciendo, imitando y repitiendo una y otra vez las delicadas labores de la actividad, viendo hacer al otro. Se enseña a investigar mostrando cómo, corrigiendo, sugiriendo, orientando. Investigar no es tanto una cuestión de definiciones sino la puesta en acción de un conjunto de saberes prácticos y operativos.

La enseñanza de saberes prácticos nos ubica en el filo entre la teoría y la práctica. El saber hacer es un saber que supera el nivel conceptual que guía 
y regula el actuar, orienta la operación. No está ajeno al mismo saber; es su misma prolongación: es el saber haciéndose. Lejos de constituir un pragmatismo vacío, es un actuar ilustrado, reposado: un operar reflexivo. Quien enseña pone en juego esa operación cada vez que indica cuándo una idea no está claramente expresada, cuando orienta lecturas, cuando brinda explicaciones teóricas, cuando muestra cómo hacer de otra manera lo que los grupos han previsto, mostrando los beneficios de sus sugerencias... O cuando se interroga, en compañía de los y las estudiantes, acerca de las razones de tomar una u otra decisión estratégica. En síntesis, cuando abre su experiencia y también su propia inexperiencia, para ponerlas a disposición de aquéllos cuyos procesos acompaña.

Por fin, como bien lo puntualiza Sánchez Puentes (2014), enseñar a investigar es, además, hacer visibles los significados y valores últimos del quehacer científico. El por qué o los para qué de carácter ético y político de la generación de conocimiento son también parte de una propuesta pedagógica. No debe omitirse la pregunta sobre los valores: la defensa de los derechos humanos, el espíritu crítico y el carácter eminentemente emancipador y libertario de la actividad, ya que "... a la acción de describir lo que se presenció se suma, así, la responsabilidad de narrar cómo llegó a ser, para contribuir a la tarea pendiente de imaginar cómo podría el mundo llegar a ser de otro modo."(Rockwell, 2009; p. 203).

\section{Notas bibliográficas}

(1) Hacer investigación cualitativa en el campo de la educación supone un esfuerzo por comprender el origen y la continuidad histórica de ciertas prácticas escolares con el objeto de procurar su transformación. Implica historizar el concepto de cultura escolar, poniendo en evidencia la coexistencia de saldos residuales y prácticas emergentes que se entrelazan con las tendencias dominantes de un momento histórico.

(2) La etnografía, considerada como enfoque o perspectiva, procura privilegiar la voz de los otros, para documentar lo no documentado de la realidad social. Su producto es una descripción densa a la que se arriba desde una posición epistemológica que presupone un trabajo teórico previo que la posibilita, y ubica al etnógrafo como sujeto social que participa de la cotidianeidad de un grupo; sujeto abierto a sus maneras de comprender el mundo y respetuoso del valor de sus conocimientos.

(3) En relación con la investigación, la hermenéutica rompe con la idea positivista del investigador como científico social, tal como lo concebía Comte, para considerarlo un intérprete de la sociedad, alguien que analiza una realidad cargada de significados y la interpreta. En esta interpretación es el lenguaje el que se convierte en vehículo de la atribución de significados, y por consecuencia, en constructor de la realidad.

(4) La noción de vigilancia epistemológica está vinculada a la preocupación que guarda 
el investigador por la coherencia teórica de su propia línea de pensamiento. Está orientada a identificar el error en su propia práctica investigativa, para buscar y aplicar, dentro de su propio marco teórico, las estrategias metodológicas que le permitan subsanarlo.

(5) La vía artesanal supone privilegiar el aprendizaje de la investigación en un entorno de taller, tal como fue concebido desde la Baja Edad Media, con el surgimiento de los gremios en las ciudades: se trata de un contexto en el que el aprendiz se apropia gradualmente de un oficio o arte, haciendo bajo la tutela de un maestro, y alcanzando grados crecientes de destreza hasta la completa autonomía.

\section{Referencias bibliográficas}

- Bergmann, G. (1953). Logical Positivism, Language, and the reconstruction of Metaphysics. In G. Bergmann. Collected Works Vol. I: Selected Papers I, edited by E. Tegtmeier, Frankfurt/Lancaster: Ontos-Verlag, 2003.

- Derrida, J. (1989). La escritura y la diferencia. Barcelona: Ed. Anthropos.

- Foucault, M. (1997). Las palabras y las cosas. Una arqueología de las ciencias humanas. Buenos Aires: Siglo XXI.

- Gadamer, H. G. (1998). El giro hermenéutico. Madrid: Editorial Cátedra.

- Geertz, C. (1987). La interpretación de la cultura. Barcelona: Gedisa.

- Guber, R. (2013). La articulación etnográfica. Descubrimiento y trabajo de campo en la investigación de Esther Hermitte. Buenos Aires: Biblos.

- Ricoeur, P. (2003). El conflicto de las interpretaciones. Ensayos de hermenéutica, 3 volúmenes: I. Hermenéutica y psicoanálisis, II. Hermenéutica y estructuralismo, III. Introducción a la simbólica del mal, Buenos Aires: Fondo de Cultura Económica.

- Rockwell, E. (2009). La experiencia etnográfica. Historia y cultura en los procesos educativos. Buenos Aires: Paidós.

- Rorty, R. (1990). El giro lingüístico: dificultades metafilosóficas de la filosofía lingüística, Buenos Aires: Paidós.

- Scribano, A. (2008). Introducción. En El proceso de investigación social cualitativa. Buenos Aires: Prometeo Libros.

- Vattimo, G. (1995). Más allá de la interpretación. Buenos Aires: Paidós.

- Zamero, M. (2013). Funciones de la escritura en el proceso de investigación. Presentación en diapositivas. Taller Metodológico a equipos seleccionados en la Convocatoria 2011: Conocer para incidir en las prácticas. Buenos Aires, INFOD.

\section{Páginas web}

- Sanchez Puentes, R. (2014) Enseñar a investigar. Una didáctica nueva de la investigación en ciencias sociales y humanas. México, IISUE/UNAM. Disponible en: http://132.248.192.241/ editorial/wpcontent/uploads/2014/10/Ense\%C3\%B1ara-investigar.pdf. Consultado el 20/06/2016. 